\title{
Brittle failure criteria of CNRB specimen for fracture toughness test
}

\author{
Manato KANESAKI*, Kiyotaka OBUNAI**, Tadao FUKUTA*, \\ Yoshihito KUROSHIMA*** and Koichi OZAKI* \\ * Department of System Engineering, Okayama Prefectural University \\ 111 Kuboki, Sojya-shi, Okayama 719-1197, Japan \\ E-mail: manato_k@cse.oka-pu.ac.jp \\ ** Department of Mechanical and Systems Engineering, Doshisya University \\ 1-3 Tataramiyakodani, Kyotanabe-shi, Kyoto 610-0394, Japan \\ *** Department of Mechanical and Control Engineering, Kyushu Institute of Technology \\ 1-1 Sensui-cho, Tobata-ku, Kitakyusyu-shi, Fukuoka 804-8550, Japan
}

Received: 28 November 2019; Revised: 9 March 2020; Accepted: 14 May 2020

\begin{abstract}
This paper proposes a technique to determine the dimensions of a CNRB specimen to be used for plane fracture toughness tests. First, the brittle fracture behavior satisfying the small-scale yielding condition is investigated through an elasto-plastic analysis. The analysis parameters are the shape parameters such as the notch ratio of the specimen based on the parallel part diameter and notch bottom diameter; tip radius; and notch angle. Based on the analysis results obtained, a fracture toughness test is conducted on a JIS-S45C (quenched and tempered) specimen to evaluate the small-scale yielding condition. The experimental results confirm a linear relationship between the square of the value obtained by dividing the plane-strain fracture toughness by the yield stress and specimen diameter. Further, the diameter of the CNRB specimen satisfying the small-scale yielding condition varies with the shape parameters. With these results, a function that can determine the CNRB specimen diameter at which brittle failure occurs under uniaxial tensile loading is proposed and validated.
\end{abstract}

Keywords : Fracture toughness, J integral, FEM, Small-scale yielding conditions

\section{Introduction}

In the manufacturing of safe and reliable structures, their design should be reviewed not only in terms of the material strength, but also in terms of the fracture mechanics. In fracture mechanics, a fracture is said to occur when the stress distribution around a crack, i.e., the stress intensity factor, reaches the plane-strain fracture toughness $K_{I C}$ (Kobayashi, 1978; Murakami, 2018). The $K_{I C}$ values of materials are typically measured and validated in accordance with industrial standards such as JIS (JIS G0564, 1999), ASTM (ASTM E1820-18ae, 2018), and ISO (ISO12737:2010, 2010). However, owing to restrictions related to the materials and/or test piece production, these tests cannot always be performed as per the prescribed standard (Nakasa and Takei, 1976). Hence, fracture toughness tests are conducted using easily manufacturable round bar specimens as alternatives to the conventional test methods (Miyata et al., 1987). In particular, Benthem et al. investigated a round bar specimen with a circumferential notch in the parallel part (hereinafter referred to as the circumferentially notched round bar (CNRB) specimen), and presented a theoretical solution for the stress intensity factor at the bottom of the notch (Benthem et al., 1973). Based on their solutions, several studies have reported the application of a fracture toughness test using a CNRB specimen under tensile loading (Noda and Takese, 2003; Savruk and Kazberuk, 2006). However, to determine the fracture toughness using the CNRB specimen, several factors should be considered. First, because the solution proposed by Benthem et al. is based on elasto-dynamics calculations, the radius at the crack tip should be set to zero. This requires introducing a fatigue pre-crack at the machined notch tip. Compared to standardized specimens, such as the compact tension (CT) specimen, the measurement and control of the fatigue crack length is difficult 
in the CNRB specimen. Moreover, they did not provide a method to determine the dimensions of the CNRB specimen. Therefore, it is necessary to develop a fracture toughness test method that does not involve introducing fatigue cracks. Otsuka et al. reported that the stress triaxiality affects the ductile fracture behavior when the notch bottom has a finite radius, as in the CNRB specimen (Otsuka et al., 1985). To obtain the fracture toughness, the specimen in the test should undergo a brittle fracture, instead of a ductile fracture. The brittle fracture of a material is governed by the energy release rate required for crack propagation (Ikeda, 1965). In this study, we focused on this energy release rate and studied the dimensions and shape corresponding to which a CNRB specimen undergoes a brittle fracture.

The objective of this study was to develop an effective method to obtain the fracture toughness of materials using the CNRB specimen. First, we conducted a conventional fracture toughness test using a CT specimen as the reference. Second, an elasto-plastic analysis was performed to investigate the effect of the dimensions of the CNRB specimen on the allowable energy release rate at which the small-scale yielding condition is satisfied. Based on the calculation results, an actual CNRB specimen was prepared to conduct a fracture toughness test, and the results were compared with those of the CT specimen. Finally, equations to determine the dimensions of the CNRB specimen were derived.

\section{Materials and methods}

\subsection{Material and heat treatment conditions}

A piece of medium carbon steel, JIS-S45C, was selected as the test material. Table 1 lists the chemical composition of this steel. It was first subjected to thermal refining to eliminate any residual strain due to the manufacturing process. After this treatment, the material was quenched and tempered to obtain a specified hardness of HV 550. The following conditions for the treatment were determined by conducting experiments. The treatment temperature for the quenching was set to $850{ }^{\circ} \mathrm{C}$ and maintained for $3600 \mathrm{~s}$, and the material was subsequently water cooled. The treatment temperature for tempering was set to $290{ }^{\circ} \mathrm{C}$ and maintained for $3600 \mathrm{~s}$. The material was then gradually cooled in the furnace. The uniformity of the heat treatment was verified by measuring the hardness distribution of a cross section of the treated material.

Table 1 Chemical composition of material. (mass, in\%).

\begin{tabular}{ccccc}
\hline $\mathrm{C}$ & $\mathrm{Si}$ & $\mathrm{Mn}$ & $\mathrm{P}$ & $\mathrm{S}$ \\
\hline 0.46 & 0.27 & 0.74 & 0.0015 & 0.02 \\
\hline
\end{tabular}

\subsection{Mechanical properties of the material}

\subsubsection{Tensile test method}

The mechanical properties of the material under uniaxial tensile loading were in accordance with JIS-Z2201. The heat-treated material was machined to the form of a standard specimen (JIS-14A) on a lathe. An axial displacement was applied to the specimen until fracture at a constant strain rate of $1.6 \times 10^{-3}\left(\mathrm{~s}^{-1}\right)$ with respect to the gauge length. At least five specimens were tested to evaluate the mechanical properties of the material.

\subsubsection{Fracture toughness test method using CT specimen}

The Mode I fracture toughness at the plane-strain state of the material was confirmed using a CT specimen in accordance with the JIS-G0564 standard, which is widely used in the industry. Figure 1 shows a schematic of the CT specimen used in this study. The width $W$ and thickness $B$ of the CT specimen were set to 30 and $7.5 \mathrm{~mm}$, respectively. The difference between the width of the specimen and the length of the crack $a$-called the ligament length-is decided by

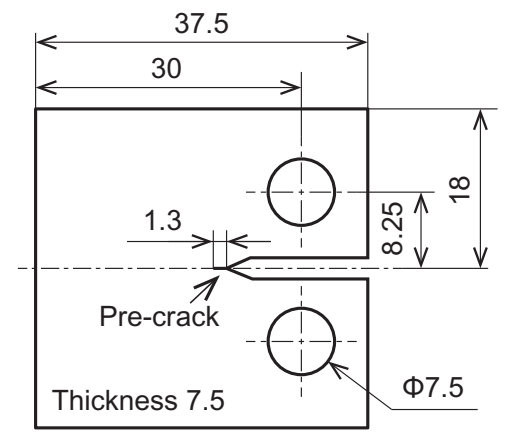

Fig. 1 Configuration of CT specimen. 
the following equation:

$$
W-a>2.5\left(\frac{K_{I C}}{\sigma_{y}}\right)^{2}
$$

Here, $K_{I C}$ and $\sigma_{y}$ denote the plane strain Mode I fracture toughness and yield strength of the material, respectively. In this study, the value of $K_{I C}$ was obtained by referring to the study by previous researchers (Ishihara and Kimura, 1986), while the value of $\sigma_{y}$ was obtained from the tensile test to decide the dimensions of the specimen. As per the standard, a $1.3 \mathrm{~mm}$-long fatigue pre-crack was introduced at the tip of the notch. After introducing the pre-crack, a tensile load was applied to the specimen at a rate of $1 \mathrm{~mm} / \mathrm{min}$ using a universal testing machine (Shimadzu, AG-X $100 \mathrm{kN}$ ). The Mode I fracture toughness at the plane-strain state of the material was calculated using the following equations:

$$
\begin{aligned}
& \xi^{\prime}=\left(\frac{a}{W}\right) \\
& K_{I C}=\frac{P_{Q}}{B W^{\frac{1}{2}}} f\left(\xi^{\prime}\right) \\
& f\left(\xi^{\prime}\right)=\left(2+\xi^{\prime}\right) \frac{0.866+4.64 \xi^{\prime}-13.32 \xi^{\prime 2}+14.72 \xi^{\prime 3}-5.6 \xi^{\prime 4}}{\left(1-\xi^{\prime}\right)^{\frac{3}{2}}}
\end{aligned}
$$

Here, $P_{Q}$ denotes the maximum applied load. The fractured surface after the test was observed using SEM. At least five specimens were tested to evaluate the fracture toughness of the material.

\subsubsection{Mechanical properties}

Figure 2(a) shows the stress-strain curve obtained from the tensile test. The Young's modulus and yield stress were approximately $210 \mathrm{GPa}$ and $1410 \mathrm{MPa}$, respectively. Figure 2(b) shows the load-crack tip opening displacement (CTOD) diagram obtained from the CT test. Figure 3 shows the fracture surface after the test. The fracture surface is brittle, and $K_{I C}$ obtained using Eq. (3) is approximately $50 \mathrm{MPa} \sqrt{\mathrm{m}}$. Table 2 lists the values of the mechanical properties. These values were used for the subsequent FEM analysis.

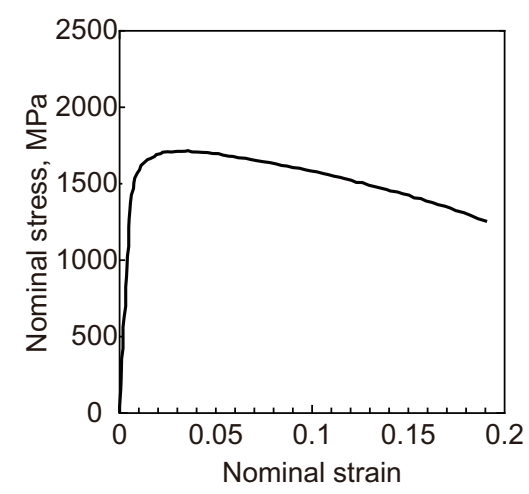

(a) Stress-Strain Curve

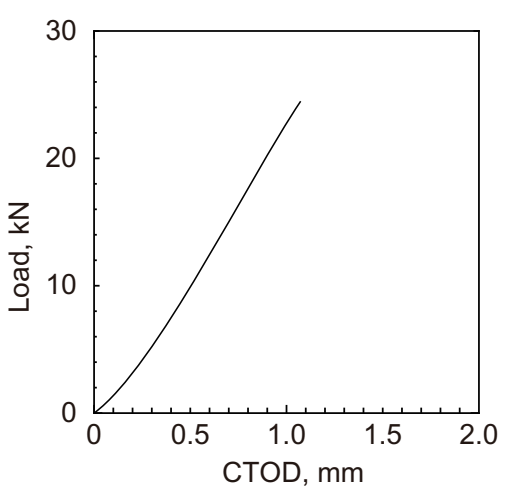

(b) Load-CTOD diagram

Fig. 2 Result of mechanical properties test.

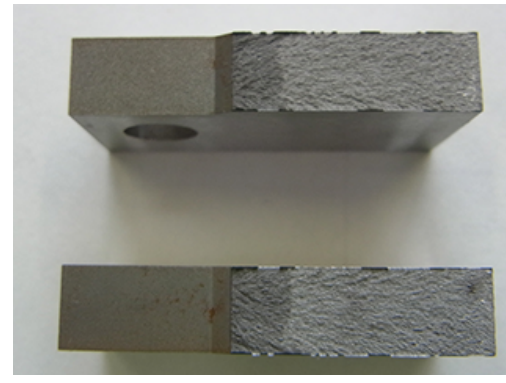

(a) Appearance

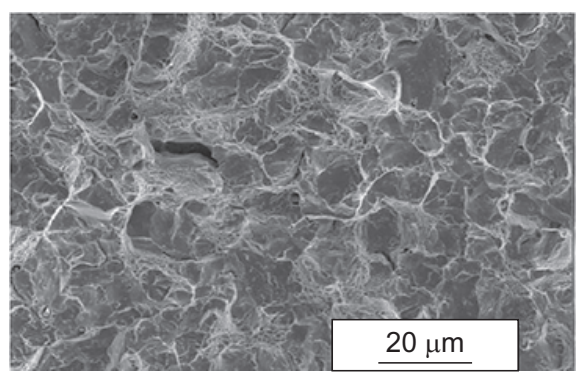

(b) SEM observation

Fig. 3 Result of fracture surface observation. 
Table 2 Material constant of S45C.

\begin{tabular}{rlc}
\hline Young' s modulus & $(\mathrm{GPa})$ & 210 \\
Poisson's ratio & & 0.3 \\
Yield stress & $(\mathrm{MPa})$ & 1410 \\
Mode I fracture toughness & $(\mathrm{MPa} \sqrt{\mathrm{m}})$ & 50 \\
\hline
\end{tabular}

\subsection{Fracture toughness test using the CNRB specimen}

\subsubsection{Analysis of the CNRB specimen}

The stress state at the notch root of the CNRB specimen was determined using the commercial elasto-plastic FEM software ABAQUS (version 6.13-5). To reduce the calculation cost, a 2D axisymmetric 1/2 model of the CNRB specimen was prepared. Figure 4 shows the calculated model. A uniform pressure load was applied to the parallel part of the specimen along the y axis, while the middle part of the specimen was constrained along this axis to simulate a symmetric condition. A fine mesh was generated around the notch. By varying the geometric conditions, 1,120 types of calculation models were prepared by following Table 3. Table 2 and Figure 5 present the material constants and nominal stress-plastic strain diagram used for the calculation, respectively.

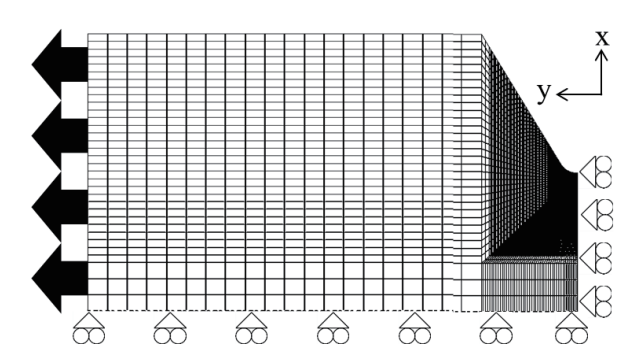

(a) Oberall view

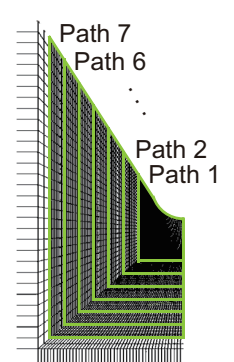

(b) Deatail of notch and path used J-integral

Fig. 4 Analysis model.

Table 3 Analysis condition list.

\begin{tabular}{cccc}
\hline $\begin{array}{c}\text { Tip radius } r \\
(\mathrm{~mm})\end{array}$ & $\begin{array}{c}\text { Notch angle } \theta \\
\left({ }^{\circ}\right)\end{array}$ & $\begin{array}{c}\text { Notch ratio } \xi \\
(-)\end{array}$ & $\begin{array}{c}\text { Parallel part diameter } D \\
(\mathrm{~mm})\end{array}$ \\
\hline 0.01 & 30 & 0.25 & 10 \\
0.1 & 60 & 0.30 & 20 \\
0.5 & 90 & 0.40 & 30 \\
1.0 & 120 & 0.50 & 40 \\
& & 0.60 & 50 \\
& & 0.70 & 60 \\
& & 0.75 & 70 \\
& & & 80 \\
& & & 90 \\
\hline
\end{tabular}

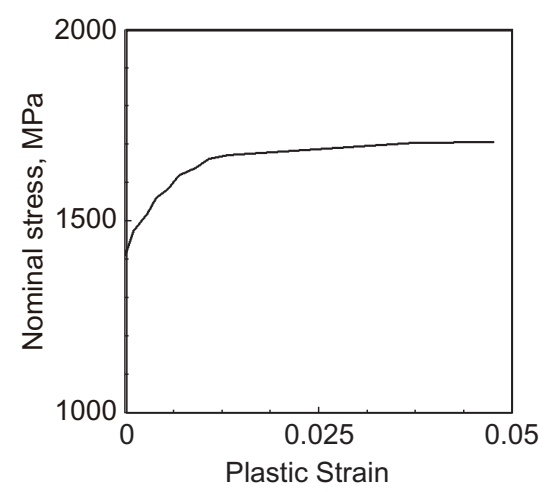

Fig. 5 Nominal stress - Plastic strain curve. 
Previous researchers (Miyata et al., 1977; Nishimura et al., 1987) suggested that when the stress state around the notch satisfies the small-scale yielding state,the stress intensity factor can be calculated from the stress distribution around the crack calculated using the FEM. However, previous studies did not discuss the criteria for the small-scale yielding state in detail. An alternative measure of the stress intensity factor is the energy release rate around the crack, which can be used to evaluate the energy state during crack propagation even if the material yields. A comparison of the energy release rate obtained by considering only the elastic behavior $g_{E}$ and that obtained by considering the elasto-plastic behavior $g_{E P}$ shows that the latter is higher because of the energy released on yielding. Therefore, if the energy release rates from the elastic and elasto-plastic analyses are largely similar, the material is expected to satisfy the small-scale yielding condition. Figure 6 shows a typical example of the calculated energy release rate obtained using the J-integral technique with respect to the applied load of the CNRB specimen model. In this study, the J-integrals are calculated along seven paths, as shown in Figure 4(b), and then averaged for the evaluation. The energy release rates obtained from the two analyses are largely the same when the applied load is small. However, when the load exceeds $2.1 \mathrm{kN}$, the energy release rates are different. This indicates that until a load of $2.1 \mathrm{kN}$, the specimen satisfies the small-scale yielding condition. Hereinafter, the energy release rate at which the small-scale yielding condition is satisfied is defined as the allowable energy release rate $g_{a l}$. In this study, $g_{a l}$ was identified through the following procedure:

A. Check if $g_{E_{i}}<g_{E P_{i}}(i$ : calculation step)

B. Calculate, at every load increment, the deviation rate $E_{S_{i}}$, defined as $\left(g_{E_{i}} / g_{E P_{i}}\right) \times 100 \%$

C. If $E_{S_{i+1}}-E_{S_{i}}$ exceeds $0.7 \%$, take $g_{E_{i}}$ as $g_{a l}$

The relationship between the geometric parameters of the CNRB specimen and $g_{a l}$ was investigated by calculating the energy release rate with the CNRB specimen models in which the geometric parameters vary. Notably, if the material satisfies the small-scale yielding condition, the energy release rate under Mode I can be converted to Mode I fracture toughness at the plane-strain state using the following equation (Kobayashi, 1978):

$$
g_{I C}=\frac{1-v^{2}}{E} K_{I C}^{2}
$$

Here, when the energy release rate exceeds the critical energy release rate $g_{I C}$, the crack propagates. $g_{a l}$ is defined as the energy release rate at which the material does not undergo plastic deformation. Therefore, if $g_{a l} \geqq g_{I C}$, the crack is considered to propagate without plastic deformation, and a brittle fracture is said to have occurred. In this study, $g_{I C}$ was obtained using Eq. (5) and the test results of the material properties. Further, $g_{a l}$ was obtained from the analysis results. If $g_{I C}=g_{a l}$, the CNRB specimen with a geometrical shape obtained from the analysis result should break because of its brittleness without undergoing any plastic deformation.

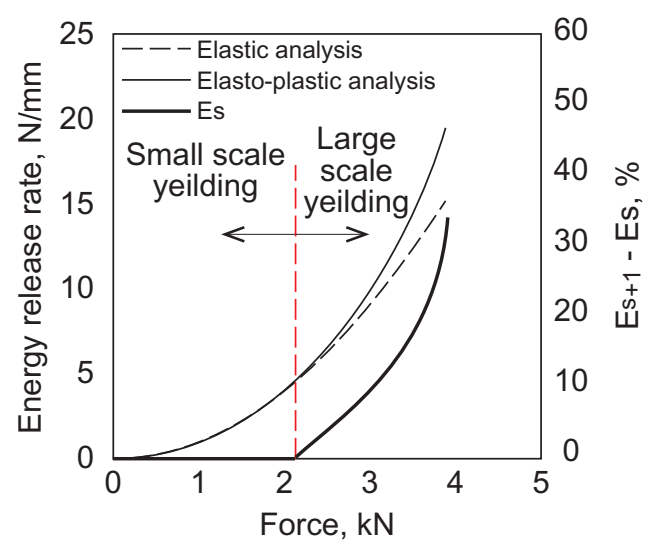

Fig. 6 A typical example of calculated energy release rate by J-integral technique with respect to applied load of CNRB specimen model.

\subsubsection{Experiment with CNRB specimen}

Figure 7 shows the configuration of the CNRB specimen prepared in this study. The heat-treated material was machined to the form of a standard specimen on a lathe. The diameter of the parallel part $D$ and notch root diameter $d$ were determined from the analysis results mentioned above. Within the limits of the lathe tools, the angle of the notch $\theta$ and tip radius $r$ were set to $60^{\circ}$ and $0.1 \mathrm{~mm}$, respectively. An axial displacement was applied to the specimen until fracture at a constant crosshead speed of $1.5 \mathrm{~mm} / \mathrm{min}$. During the test, the notch of the specimen was observed using a CCD camera (BITRAN, BH-53L) to measure the CTOD. Figure 8 shows an image of the notch obtained using the 


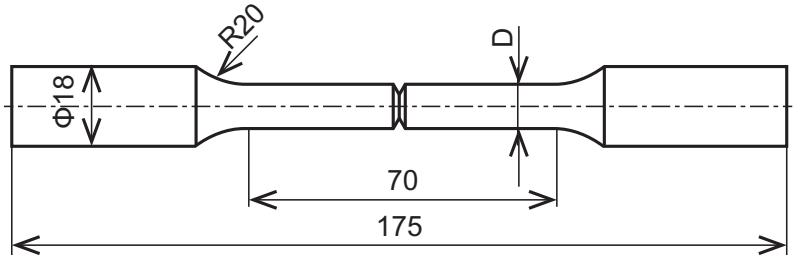

(a) Oberall view

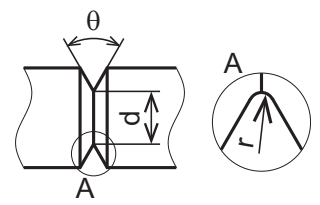

(b) Deatail of notch

Fig. 7 Configuration of round bar with circumferential notch.

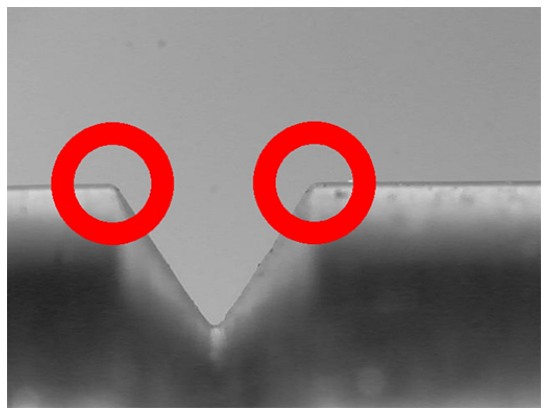

(a) Before test

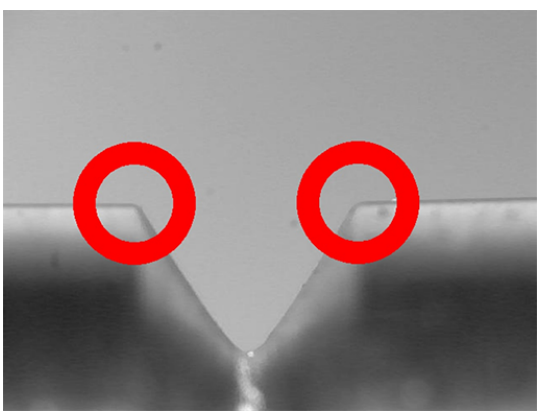

(b) Before fracture

Fig. 8 Tracking point of CCD image.

CCD camera. The edge indicated by the red circle in the figure is measured as the tracking point. The start of the data acquisition and the start of the image capturing process were synchronized, and their sampling periods were set to 100 and $1000 \mathrm{~ms}$, respectively. A minimum of five specimens were tested.

\section{Results and discussion}

\subsection{Fracture toughness test}

Figure 9(a) shows the typical results of $g_{a l}$ with respect to the diameter of the parallel part of the CNRB specimen. Here, the angle $\theta$ and tip radius $r$ are fixed at $60^{\circ}$ and $0.1 \mathrm{~mm}$, respectively. The results suggest that the allowable energy release rate increases with an increase in the diameter of the CNRB specimen owing to the increment in the ligament length. For a Mode I fracture toughness of $50 \mathrm{MPa} \sqrt{\mathrm{m}}$ at the plane-strain state obtained from the CT test, a Young's modulus of $210 \mathrm{GPa}$, and a Poisson's ratio of 0.3 obtained from the tensile test, the energy release rate is calculated to be $10.8 \mathrm{~N} / \mathrm{mm}$ using Eq. (5). With this and the calculated results, it is suggested that the diameter of the CNRB specimen should be greater than $13.9 \mathrm{~mm}$. Two CNRB specimens with different parallel part diameters (12 and $14 \mathrm{~mm})$ but with the same tip radius, notch angle, and notch ratio were prepared and tested. Figure 10 shows the typical load-CTOD diagrams of the CNRB specimens. When the specimen diameter is smaller than the estimated value $(D=12 \mathrm{~mm})$, the

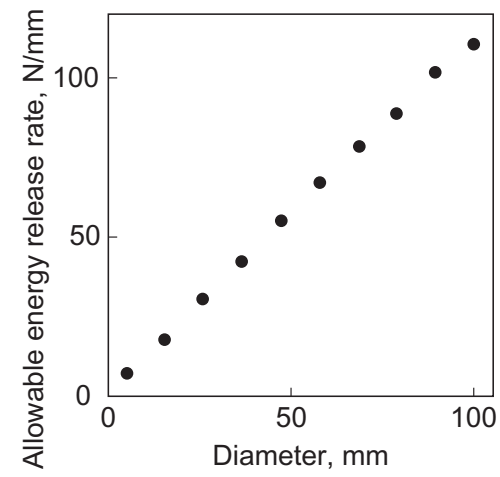

(a) Typical calculated results

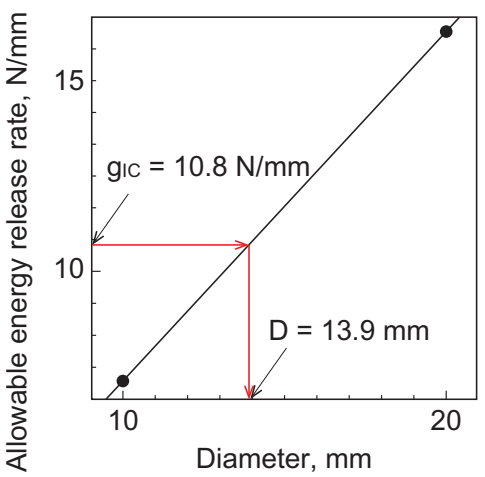

(b) Determination of diameter for brittle fracture

Fig. 9 Energy release rate-Diameter (S45C, $r=0.1 \mathrm{~mm}, \theta=60^{\circ}, \xi=0.4$ ). If the shape conditions of the tip radius, notch angle, and notch ratio obtained on machining the specimen are given, the estimated $K_{I C}$ value of the member and the specimen diameter satisfying the small-scale yielding condition according to each parameter will change. 


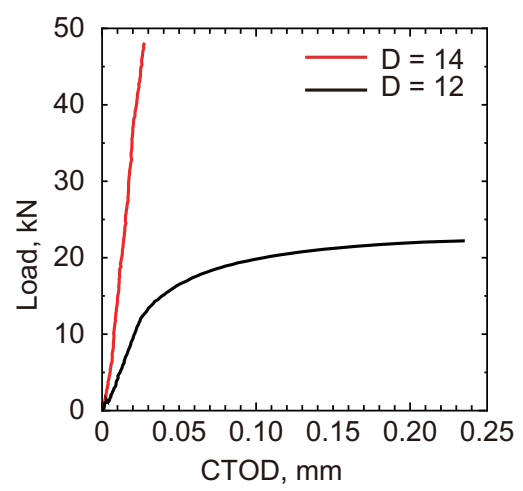

(a) Load-CTOD curve

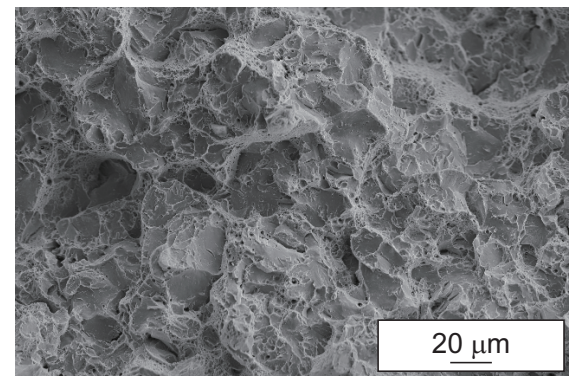

(b) Fracture surface at $D=14$

Fig. 10 Comparison of the Load-CTOD curve between specimens $D=12$ and $D=14$ : Plastic deformation occurs in Specimen $D=12$. On the other hand, specimen $D=14$ exhibits linear behavior until final failure. As a result of the fracture surface observation, the $D=14$ specimen suffers a brittle fracture.

load gradually increases with an increase in the CTOD because of the plastic deformation. On the other hand, when the specimen diameter is greater than the estimated value $(D=14 \mathrm{~mm})$, the load increases linearly until fracture. These results point to a brittle fracture of the CNRB specimen, even when the fatigue pre-crack is not introduced. Moreover, this demonstrates that the calculation results obtained using the proposed method can be used to effectively predict the state of yielding under the specified load conditions.

\subsection{Derivation of a dimension determination formula}

\subsubsection{Concept underlying the dimension determination formula}

To determine the dimensions of the specimen, particularly the diameter of the parallel part, from its material constants $\left(K_{I C}, \sigma_{y}\right)$ and configurations $(r, \theta, \xi)$, the following formula for the CT specimen was used:

$$
D_{C N R B}>\alpha(r, \theta, \xi)\left(\frac{K_{I C}}{\sigma_{y}}\right)^{2}
$$

Here, the coefficient $\alpha$ is defined as a function of the configurations of the specimen. Figure 11 shows the relationship between the diameter of the CNRB specimen and the second term on the right-hand side of Eq. (6). In this figure, the tip radius $r$ and notch angle $\theta$ are fixed at $0.1 \mathrm{~mm}$ and $60^{\circ}$, respectively. The calculated results suggest that under certain shape conditions and under all notch ratio conditions, there is a linear relationship between the specimen diameter, in cases where the small-scale yielding condition is observed, and the ratio of the fracture toughness to the yield stress. Under this condition, the shape factor $\alpha$ is the slope of the straight line representing the same shape condition in the figure. Therefore, when the three variables $r, \theta$, and $\xi$ are defined, the specimen diameter can be obtained as the coefficient for which the small-scale yielding condition represented by the ratio of the estimated $K_{I C}$ to yield stress is satisfied. Accordingly, the coefficient $\alpha$ is defined as the function of the configurations of the specimen and is represented by the product of each

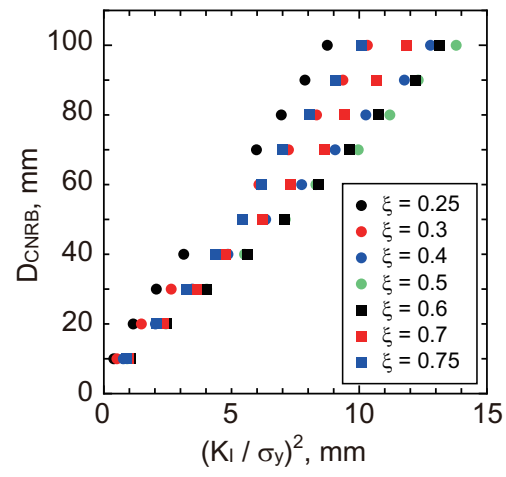

Fig. 11 Relationship between the diameter of the CNRB specimen with respect to the second term on the right side of Eq. (6). The calculated results suggest that under certain shape conditions and under all notch ratio conditions, there is a linear relationship between the specimen diameter, in cases where the small-scale yielding condition is observed, and the ratio of the fracture toughness to the yield stress. 
shape function as follows:

$$
\alpha(r, \theta, \xi)=\alpha_{1}(\xi) \alpha_{2}(\theta) \alpha_{3}(r)
$$

\subsubsection{Effect of notch ratio on the coefficient}

Figure 12 shows the calculated results of the coefficient $\alpha$ with respect to the notch ratio under different tip radius conditions. Comparing the calculated results for the same notch ratio, we find that the coefficient $\alpha$ increases with an increase in the tip radius. This means that if the tip radius is large, the diameter of the CNRB specimen would be large because of the large-scale yielding. Moreover, the coefficient $\alpha$ calculated from the shape function has a relatively strong dependency on the tip radius under all notch angle conditions. From these results, the shape function related to the notch ratio was approximated using the following polynomial equation:

$\alpha_{1}(\xi)=a \xi^{4}+b \xi^{3}+c \xi^{2}+d \xi+e$

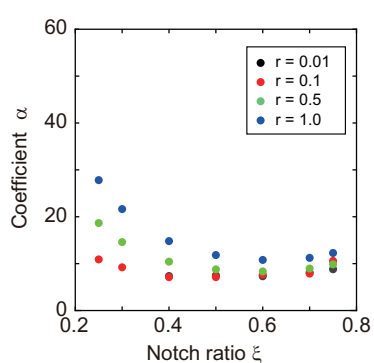

(a) $\theta=30^{\circ}$

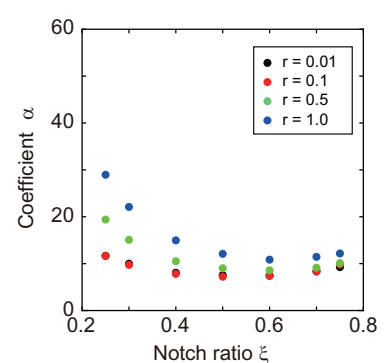

(b) $\theta=60^{\circ}$

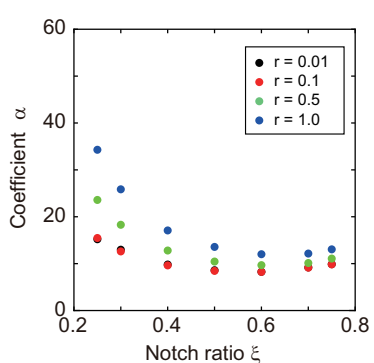

(c) $\theta=90^{\circ}$

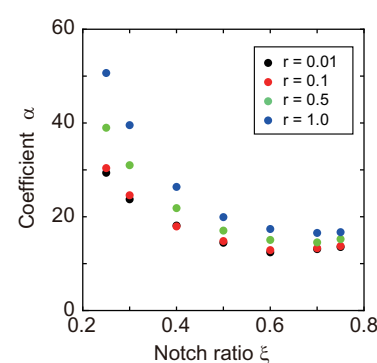

(d) $\theta=120^{\circ}$

Fig. 12 Relationship between the coefficient and notch ratio. The coefficient $\alpha$ calculated from the shape function has a relatively strong dependency on the tip radius under all notch angle conditions.

\subsubsection{Effect of notch angle on the coefficient}

Figure 13 shows the calculated results of the coefficient $\alpha$ with respect to the notch angle under different notch ratio conditions. The results show that the coefficient increases with an increase in the notch angle, even when the notch ratio and tip radius are varied. The shape function for the notch angle is approximated using the following equation:

$$
\alpha_{2}(\theta)=g(\xi)(\sinh (\theta))^{h}+i
$$

Here, the coefficient of the function $g(\xi)$ is considered, because if the notch ratio is given, the relationship between the shape function and notch angle shows almost the same tendency. The function $g(\xi)$ is approximated using the following polynomial equation:

$$
g(\xi)=j \xi^{5}+k \xi^{4}+l \xi^{3}+m \xi+n \xi+p
$$

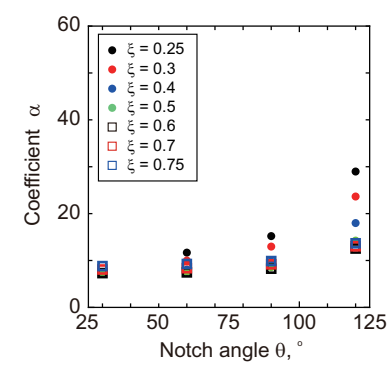

(a) $r=0.01$

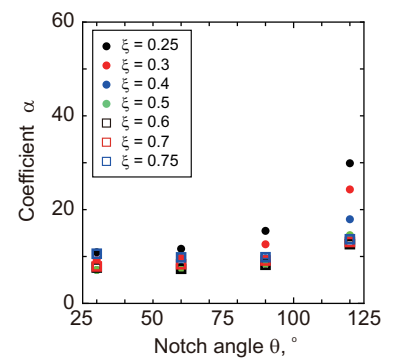

(b) $r=0.1$

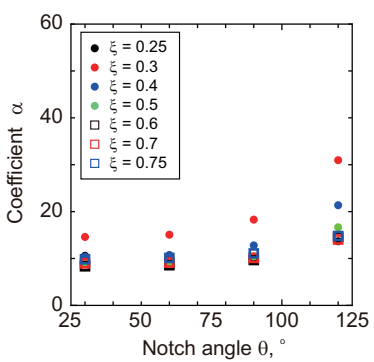

(c) $r=0.5$

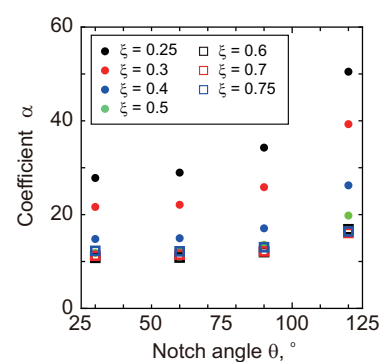

(d) $r=1.0$

Fig. 13 Relationship between the coefficient and notch angle. The coefficient $\alpha$ increases with an increase in the notch angle, even when the notch ratio and tip radius are varied.

\subsubsection{Effect of tip radius on the coefficient}

Figure 14 shows the calculated results of the coefficient $\alpha$ with respect to the inverse of the tip radius under different notch angle conditions. The results show that the coefficient decreases rapidly with an increase in the inverse of the tip radius. The decreasing trend almost plateaus when $1 / r$ exceeds 10 . The shape function related to the tip radius is approximated using the following equation:

$$
\alpha_{3}(r)=q r+s
$$




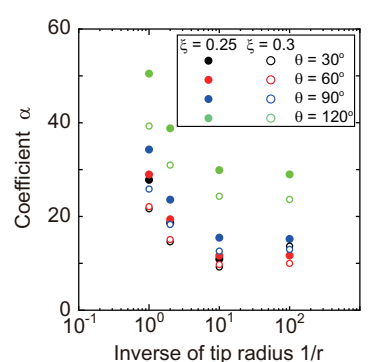

(a) $\xi=0.25 \& 0.3$

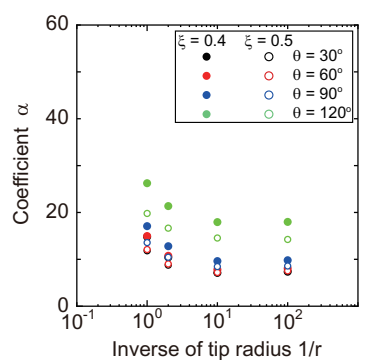

(b) $\xi=0.4 \& 0.5$

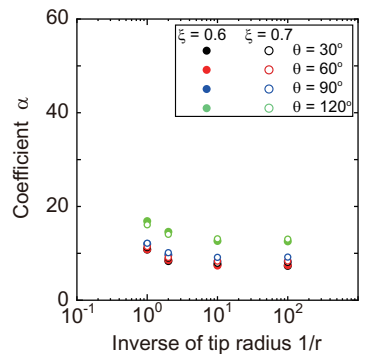

(c) $\xi=0.6 \& 0.7$

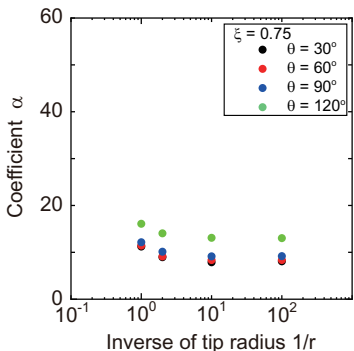

(d) $\xi=0.75$

Fig. 14 Relationship between the coefficient and inverse of tip radius. The coefficient $\alpha$ decreases rapidly with an increase in the inverse of the tip radius. However, the decreasing trend almost plateaus when $1 / r$ exceeds 10.

\subsection{Estimated results}

Based on the above considerations, a regression analysis was conducted to examine the parameters of the dimension determination formula. The estimation formula was examined by optimizing the coefficient of determination $R^{2}$. Figure 15 shows the typical analysis results of the shape factor $\alpha$ obtained using the form function estimation equation determined from the regression analysis, represented as a solid line. Now, $R^{2}=0.956$ may not match under all conditions. The shape factor calculated from the analysis results is $\alpha_{J}$ and that calculated from the estimation formula is $\alpha_{F}$. The matching rate $E=\alpha_{J} / \alpha_{F}$ is examined. The matching rate is $90 \%$ or less for 63 of the 110 conditions. Therefore, we focused on the remarkable change in the shape factor for tip radii between $r \leq 0.1$ and $r>0.1$. The specimen size determination formula was divided into two parts to determine the shape function constants. Figure 16 shows the results. Table 4 lists the final parameters used. When $r \leq 0.1$, as shown in Fig. 16(a), $R^{2}=0.995$, and the match rate is $90 \%$ or more in all but one condition. Among these, the agreement rate in 46 of the conditions is over 95\%. Further, when $r>0.1$, as shown in Fig.

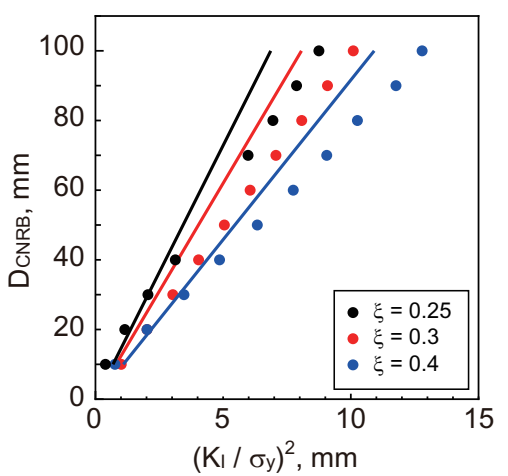

(a) $\xi=0.25 \sim 0.4$

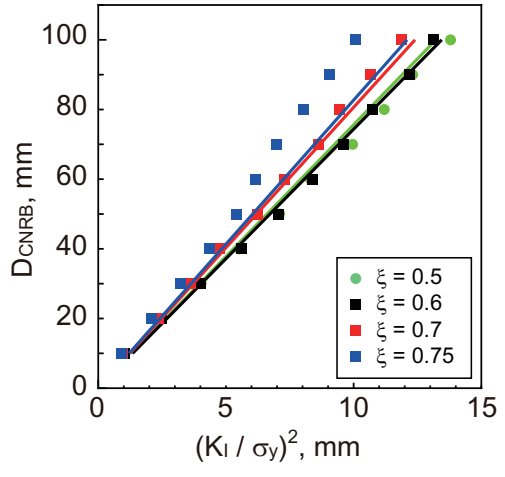

(b) $\xi=0.5 \sim 0.75$

Fig. 15 An example of the results of the regression analysis. The matching rate is $90 \%$ or less for 63 conditions out of 110 conditions.

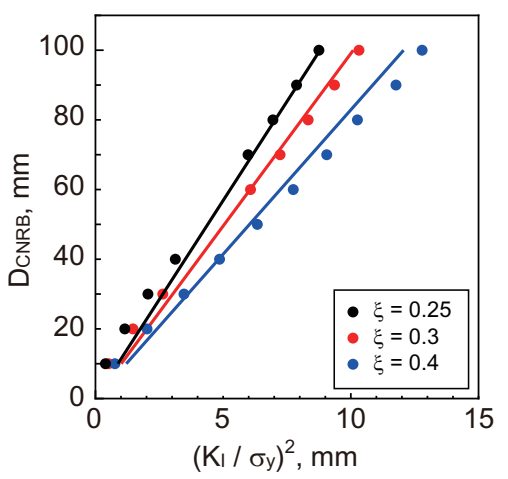

(a) $\xi=0.25 \sim 0.4$

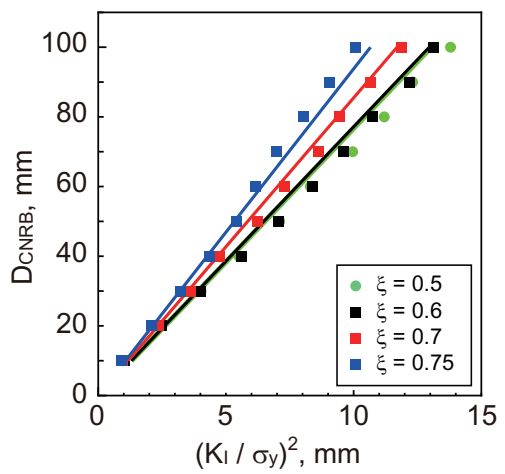

(b) $\xi=0.5 \sim 0.75$

Fig. 16 An example of the result of the regression analysis performed by dividing the chip radius. The coefficient of determination and the agreement rate improved. 
Table 4 Constants and values in equaton (8) through (11).

\begin{tabular}{cccc}
\hline Used function & Constant & $0.01 \leq r \leq 0.1$ & $0.1<r \leq 1.0$ \\
\hline$\alpha_{1}(\xi)$ & $\mathrm{a}$ & 18.416 & 23.26 \\
& $\mathrm{~b}$ & -38.81 & -54.526 \\
& $\mathrm{c}$ & 33.774 & 50.653 \\
& $\mathrm{~d}$ & -14.033 & -22.121 \\
& $\mathrm{e}$ & 3.087 & 4.384 \\
\hline$\alpha_{2}(\theta)$ & $\mathrm{h}$ & \multicolumn{2}{c}{2.390} \\
& $\mathrm{i}$ & \multicolumn{2}{c}{15.167} \\
\hline$g(\xi)$ & $\mathrm{j}$ & \multicolumn{2}{c}{-103.70} \\
& $\mathrm{k}$ & \multicolumn{2}{c}{265.65} \\
& 1 & \multicolumn{2}{c}{-261.85} \\
& $\mathrm{~m}$ & \multicolumn{2}{c}{125.01} \\
& $\mathrm{n}$ & \multicolumn{2}{c}{-30.13} \\
\hline$\alpha_{3}(r)$ & $\mathrm{n}$ & 3.48 \\
& $\mathrm{p}$ & 0.12 & 0.643 \\
& $\mathrm{q}$ & 0.78 & 0.673 \\
\hline Coefficient of determination $R^{2}$ & 0.995 & 0.985 \\
\hline
\end{tabular}

16 (b), $R^{2}=0.985$, and the matching rate is $90 \%$ or more in all but five conditions. Among them, the agreement rate for 27 of the conditions is over $95 \%$. The estimation results can be improved by dividing the shape function into two sections considering the change in the tip radius.

To investigate the validity of the proposed function, the diameter of the specimen mentioned in the previous section was calculated. Here, the material constant of the specimen was taken from Table 2, and the tip radius $r$, notch angle $\theta$, and notch ratio $\xi$ were set to $0.1 \mathrm{~mm}, 60^{\circ}$, and 0.4 , respectively. The calculated diameter $\left(D_{C N R B}=13.7 \mathrm{~mm}\right)$ shows a good agreement with the experimental results reported in Section 3.1. These results suggest that the proposed function can effectively estimate the CNRB specimen diameter at which brittle failure occurs under uniaxial tensile loading. To calculate the fracture toughness of the material using the CNRB specimen, further experiments based on this result should be conducted.

\section{Conclusions}

In this study, we examined the effectiveness and suitability of a fracture toughness test using a CNRB specimen under tensile loading. The elasto-plastic behavior of the specimen was analyzed using the FEM. The following conclusions can be drawn from the study:

(1) The state of yielding under specified load conditions can be effectively estimated by comparing the energy release rates with and without considering the elasto-plastic behavior of the material.

( 2 ) The brittle fracture of the CNRB specimen can be successfully replicated, even without introducing a fatigue pre-crack.

(3) An equation to determine the dimensions of the CNRB specimen is proposed.

\section{References}

ASTM International, E1820-18ae1 Standard Test Method for Measurement of Fracture Toughness, (2018)

Benthem, J. P. and Koiter,W., Asymptotic approximation to crack problems, Mechanics of fracture, vol.1(1973), pp.131178

Hitham M. T., Sugawara, Y. and Majima, T., Effect of notch depth on strain-concentration factor of notched cylindrical bars under static tension, European Journal of Mechanics A/Solids, Vol.24, No.5 (2005), pp.406-416

Ikeda, K., Recent Dtudies on Brittle Fracture Initiation, Journal of the Japan Welding Society, Vol.34, Issue.10 (1965), pp.1038-1048, (in Japanese)

Ishihara, M. ande Kimura, S., Effect of Austenitizing Temperature on the Fracture Toughness of SCM440 and S45C Steels, Journal of the Society of Materials Science, Japan, Vol.35, Issue.396 (1986), pp.1010-1015, (in Japanese)

International Organization for Standardization, Metallic materials - Determination of plane-strain fracture toughness, (2010)

Japanese Industrial Standard, JIS G 0564 Metallic materials-Determination of Plane-strain fracture toughness, (1999), (in Japanese) 
Li, D. M. and Bakker, A.., Fracture toughness evaluation using circumferentially-cracked cylindrical bar specimens, Engineering Fracture Mechanics, Vo.57, No.1 (1997), pp.1-11

Kameyama, H., Production method of thermal conductive catalyst, Japanese patent disclosure H00-100100 (1990)

Kobayashi, H., Fracture toughness and Its Evaluation Method (1), Transactions of JSMS, Vol.27, No.297 (1978), pp.495505 (in Japanese)

Miyata, T., Otsuka, A., Mitsubayashi, M., Haze, T. and Aihara, S., Prediction of Fracture Toughness of Steels by Local Fracture Criterion, Journal of the Society of Materials Science, Japan, Vol.37, No.419(1988), pp.897-903(in Japanese).

Miyata,H., Shida, S. and KusumotoS., Calculation method of stress intensity factor by finite element method, Transactions of the JSME, Vol.43, No.365(1977), pp.26-37 (in Japanese).

Nakasa, K. and Takei, H., The Sheet Thickness Dependency of Fracture Toughness in High Strength Steels, Tetsu-toHagane, Vol.62, No.12(1976), pp.1523-1531(in Japanese).

Noda, N. and Takase, Y., Generalized stress intensity factors of V-shaped notch in a round bar under torsion, tension, and bending, Engineering Fracture Mechanics, Vol.70, No.11(2003), pp.1447-1466.

Nishimura, A., Tatemichi, A., Miura, H. and Sakamoto, T., Life Estimation For IC Plastic Packages Under Temperature Cycling Based on Fracture Mechanics, IEEE Transactions on Components, Vol.10(1987), pp.637-642.

Otsuka, A., Miyata, T., Sakurai, T and Iida, H., Effect of Stress Triaxiality on Ductile Fracture-Material Dependence and Effect of Stress Relief Annealing-, Journal of the Society of Materials Science, Japan, Vol.34, No.381(1985), pp.622-626(in Japanese).

Savruk, M. P. and Kazberuk, A., Relationship between the stress intensity and stress concentration factors for sharp and rounded notches, Materials Science, Vol.42(2006), pp.725-738.

Shabara, M. A. N., El-Domiaty, A. A. and Al-Ansary, M. D., Estimation of plane strain fracture toughness from circumferentially bluntly notched round-bar specimens, Engineering Fracture Mechanics, Vol.54, No.4 (1996), pp.533-541.

Tagawa,T., Miyata, T. and Otsuka A., Evaluation of Cleavage Fracture Toughness by Statistical Local Fracture Criterion Approach, Journal of the Society of Materials Science, Japan, Vol.41, No.467(1992), pp.1227-1233(in Japanese). 